T. SATO

KODAI MATH. J.

4 (1981), 129-136

\title{
THE SPECTRUM OF THE LAPLACIAN FOR SOME 6-DIMENSIONAL $K$-SPACES
}

\author{
By TAKuji SATO
}

\section{Introduction.}

Let $(M, g)$ be a compact orientable Riemannian manifold with metric tensor $g$. By $\Delta$ we denote the Laplacian acting on differentiable functions on $M$. Then we have the spectrum

$$
\operatorname{Spec}(M, g)=\left\{0 \geqq \lambda_{1} \geqq \lambda_{2} \geqq \cdots>-\infty\right\}
$$

where each eigenvalue is repeated as many time as its multiplicity indicates. The spectrum $\operatorname{Spec}(M, g)$ exerts an influence on the geometry of $(M, g)$. It is interesting to see the relation of $\operatorname{Spec}(M, g)$ on the geometry of $(M, g)$. For the study of this, M. Berger and T. Sakai used the coefficients of the asymptotic expansion of Minakshisundaram-Pleijel. In [6], after a long calculation, Sakai obtained the following

THEOREM A. Let $(M, g)$ and $\left(M^{\prime}, g^{\prime}\right)$ be compact connected orrentable Einstem manifolds with dimension $M=6$. We assume that $\chi(M)=\chi\left(M^{\prime}\right)$ and $\operatorname{Spec}(M, g)=$ $\operatorname{Spec}\left(M^{\prime}, g^{\prime}\right)$ hold where $\chi(M)$ denotes the Euler-Ponncare characteristic of $M$. Then $(M, g)$ is locally symmetruc if and only if $\left(M^{\prime}, g^{\prime}\right)$ is locally symmetruc.

In the present paper, we shall prove the following

Theorem B. Let $(M, g, J)$ and $\left(M^{\prime}, g^{\prime}, J^{\prime}\right)$ be 6-dimensional complete, connected K-spaces which are non-Kählerian. We assume that $\chi(M)=\chi\left(M^{\prime}\right)$ and $\operatorname{Spec}(M, g)=\operatorname{Spec}\left(M^{\prime}, g^{\prime}\right)$. Then $(M, g)$ is Riemannian locally 3-symmetric if and only if $\left(M^{\prime}, g^{\prime}\right)$ is Riemannian locally 3-symmetruc.

It is well-known that the 6-dimensional non-Kähler $K$-space $(M, g, J)$ is an Einstein manifold with positive scalar curvature [5]. Therefore $M$ is compact by Myers' theorem. The study of Riemannian 3-symmetric space has been done by A. Gray [4]. We shall give some definitions and preliminary facts on Riemannian 3-symmetric spaces in $\S 2$. Particularly we shall show the relationship between Riemannian 3-symmetric spaces and homogeneous $K$-spaces. In $\S 3$, we shall prove Theorem $B$ by slight modification of the proof of Theorem A.

Received December 14, 1979 
The author wishes to express his hearty thanks to Prof. K. Takamatsu and Prof. K. Sekigawa for their kindly advices.

\section{Riemannian 3-symmetric spaces.}

Throughout this paper, manifolds and tensor fields are assumed to be of class $C^{\infty}$ unless otherwise specified.

Let $(M, g)$ be a Riemannian manifold with Riemannian connection $\nabla$. By $R=\left(R_{a b c}{ }^{d}\right), R_{1}=\left(R_{a b}\right)$ and $S$ we denote the Riemannian curvature tensor, the Ricci curvature tensor and the scalar curvature, respectively.

Suppose that $(M, g)$ admits a local isometry $\theta_{p}: U_{p} \rightarrow U_{p}$ for each point $p$ of $M$ such that

i) $\theta_{p}{ }^{3}=1$,

ii) $p$ is an isolated fixed point of $\theta_{p}$,

iii) the tensor field $\Theta$ defined by $\Theta_{p}=\left(d \theta_{p}\right)_{p}$ is $C^{\infty}$.

Then we can define an aimost complex structure $J$ by

$$
\frac{\sqrt{3}}{2} J_{p}=\Theta_{p}+\frac{1}{2} I_{p}
$$

where $I_{p}$ denotes the identity of $T_{p}(M)$. Since each $\theta_{p}$ is an isometry, the Riemannian metric $g$ is almost Hermitian with respect to $J$. Furthermore, we assume

iv) each $\theta_{p}$ is holomorphic with respect to $J$, i. e.,

$$
d \theta_{p} \circ=J \circ d \theta_{p} \text { on } U_{p} .
$$

Definition 1. A Riemannian manifold $(M, g)$ is called a Riemanman locally 3-symmetruc space if $(M, g)$ admits a family of local isometries $\left\{\theta_{p}\right\}$ satisfying the above conditions i), ii), iii) and iv). An almost complex structure $J$ defined by (2.1) is said to be a canonical one.

Definition 2. A Riemannian locally 3-symmetric space $(M, g)$ is called a Riemannian 3-symmetruc space if each $\theta_{p}$ can be extended to a global holomorphic isometry of $M$.

As an example, we shall consider the 6-dimensional unit sphere $S^{6}$. Let $\mathcal{C}$ be the Cayley algebra and $E$ be a set of all pure imaginary Cayley numbers. Then $E$ can be identified with the 7-dimensional Euclidean space. For any two points $x, y$ of $E$, the inner product $(x, y)$ and the vector product $x \times y$ are defined by

$$
\begin{aligned}
& -(x, y)=\text { the real part of } x y, \\
& x \times y=\text { the imaginary part of } x y,
\end{aligned}
$$

where $x y$ is a product of $x$ and $y$ in $\mathcal{C}$. The 6-dimensional unit sphere $S^{6}$ is 
the set of all $x \in E$ such that $(x, x)=1$. For any point $a$ of $S^{6}$, we define a map $\theta_{a}: S^{6} \rightarrow S^{6}$ by

$$
\theta_{a}(x)=\frac{3}{2}(a, x) a-\frac{1}{2} x+\frac{\sqrt{3}}{2} a \times x .
$$

Then we can check by straightforward computation that $\theta_{a}{ }^{3}=1$ and $a$ is an isolated fixed point of $\theta_{a}$. With this family $\left\{\theta_{a}\right\}$ and a canonical metric $g_{0}$, $\left(S^{6}, g_{0}\right)$ becomes a Riemannian 3 -symmetric space. It may be verified that the canonical almost complex structure $J_{0}$ of this family coincides with the one constructed by A. Frölicher [2], and hence $\left(S^{6}, g_{0}, J_{0}\right)$ becomes a $K$-space [3].

Definition 3. Let $(M, g, J)$ be an almost Hermitian manifold. A tensor field $T$ of type $(1,2)$ on $M$ is called a homogeneous structure if it satisfies

(a) $\left(\nabla_{X} R\right)(Y, Z)=[T(X), R(Y, Z)]-R(T(X) Y, Z)-R(Y, T(X) Z)$,

(b) $\left(\nabla_{X} T\right) Y=[T(X), T(Y)]-T(T(X) Y)$,

(c) $\nabla_{X} J=[T(X), J]$,

(d) $g(T(X) Y, Z)+g(Y, T(X) Z)=0$.

In [8], Sekigawa proved the following

THEOREM 2.1. Let $(M, g, J)$ be a homogeneous almost Hermitian manifold. Then, there exists a homogeneous structure $T$ on $M$. Conversely, if a connected, simply connected, complete almost Hermitian manifold $(M, g, J)$ admits a homogeneous structure $T$, then $(M, g, J)$ is a homogeneous almost Hermitian manfold.

Now let $(M, g, J)$ be a $K$-space. We put

$$
\hat{T}(X) Y=\frac{1}{2} J\left(\nabla_{X} J\right) Y .
$$

The tensor field $\hat{T}$ plays an important role in a $K$-space. It has been shown that $\hat{T}$ always satisfies the conditions (b), (c), (d) for the homogeneous structure [9]. Hence we shall consider only the condition (a). We define a tensor field $L$ of type $(1,4)$ by

$$
L(X, Y, Z)=\left(\nabla_{X} R\right)(Y, Z)-[\hat{T}(X), R(Y, Z)]+R(\hat{T}(X) Y, Z)+R(Y, \hat{T}(X) Z) .
$$

Obviously, $L=0$ means that the tensor field $\hat{T}$ satisfies (a). In the case of $\operatorname{dim} M=6$, Sekigawa has calculated in his paper [9] the square of the length of $L$. By $|P|^{2}$ we denote the square of the length of a tensor $P$.

THEOREM 2.2. Let $(M, g, J)$ be a complete 6-dimensional complete non-Kähler K-space. Then we have

$$
\int_{M}|L|^{2} d M=\int_{M}\left[|\nabla R|^{2}-\frac{1}{15} S\left(|R|^{2}-\frac{1}{15} S^{2}\right)\right] d M,
$$

where $d M$ denotes the volume element of $(M, g)$. 
As for the relation between Riemannian 3-symmetric spaces and homogeneous $K$-spaces, the present author [7] proved the following

THEOREM. 2.3. Let $(M, g, J)$ be a complete, connected and simply connected $K$-space. Then $(M, g, J)$ is a homogeneous almost Hermitian manıfold with homogeneous structure $\hat{T}$ if and only if $(M, g)$ is a Riemannian 3-symmetruc space with canonical almost complex structure $J$.

We shall remark that the proof of the above theorem in [7] actually yields the following slightly more precise result.

THEOREM 2.4. Let $(M, g, J)$ be a complete and connected $K$-space. Then the tensor field $\hat{T}$ is the homogeneous structure of $(M, g, J)$ if and only if $(M, g)$ is a Riemannian locally 3-symmetric space with canonical almost complex structure J.

\section{Proof of Theorem B.}

We first prove two lemmas. We put

$$
\begin{aligned}
& \hat{R}=R^{a b c d} R_{a b}{ }^{u v} R_{c d u v}, \\
& \stackrel{\circ}{R}=R^{a b c d} R_{a}{ }_{c}{ }_{c}^{v} R_{b u d v} .
\end{aligned}
$$

LEMMA 3.1. Let $(M, g)$ be a compact orientable Einstern manifold of dimension 6. Then we have

$$
\int_{M} \stackrel{\circ}{R} d M=\frac{1}{4} \int_{M}\left[|\nabla R|^{2}+\frac{1}{3} S|R|^{2}-\hat{R}\right] d M .
$$

Proof. From the computation, we get the following Lichnerowicz's formula.

$$
\begin{aligned}
\frac{1}{2} \Delta\left(|R|^{2}\right)= & |\nabla R|^{2}+4 R^{a b c d} \nabla_{a} \nabla_{c} R_{b d} \\
& +2 R^{u v} R_{u}^{a b c} R_{v a b c}-\hat{R}-4 \stackrel{\circ}{R} .
\end{aligned}
$$

If $(M, g)$ is 6-dimensional Einsteinian, (3.2) is reduced to

$$
\frac{1}{2} \Delta\left(|R|^{2}\right)=|\nabla R|^{2}+\frac{1}{3} S|R|^{2}-\hat{R}-4 \stackrel{\circ}{R} .
$$

Applying Green's theorem to (3.3), we get (3.1).

Making use of Lemma 3.1, we obtain the following formula (3.4) due to Sakai [6].

LEMMA 3.2. Let $(M, g)$ be a compact orientable Einstern manfold of dimension 6. The Euler-Poincaré characterıstıc $\chi(M)$ is given by 


$$
\chi(M)=\frac{1}{384 \pi^{3}} \int_{M}\left[6 \hat{R}-2|\nabla R|^{2}+\frac{1}{9} S^{3}-\frac{5}{3} S|R|^{2}\right] d M
$$

Proof. In a 6-dimensional compact orientable Riemannian manifold, it is well-known that $\chi(M)$ is given by

$$
\begin{aligned}
\chi(M)=\frac{1}{384 \pi^{3}} \int_{M}[ & S^{3}-12 S\left|R_{1}\right|^{2}+3 S|R|^{2}+16 R^{a b} R_{a}{ }^{c} R_{b c} \\
& +24 R^{a b} R^{c d} R_{a b c d}-24 R^{u v} R_{u}{ }^{a b c} R_{v a b c} \\
& \left.+2 \hat{R}-8 R^{a b c d} R_{a}{ }^{u}{ }_{c}^{v} R_{b v d u}\right] d M .
\end{aligned}
$$

By using the Bianchi's identity repeatedly, we get

$$
R^{a b c d} R_{a}{ }^{u}{ }^{v} R_{b v d u}=\stackrel{\circ}{R}-\frac{1}{4} \hat{R} .
$$

Thus we have

In Einsteinian case,

$$
\begin{aligned}
\chi(M)=\frac{1}{384 \pi^{3}} \int_{M}[ & S^{3}-12 S\left|R_{1}\right|^{2}+3 S|R|^{2}+16 R^{a b} R_{a}{ }^{c} R_{b c} \\
& +24 R^{a b} R^{c d} R_{a c b d}-24 R^{u v} R_{u}{ }^{a b c} R_{v a b c} \\
& -8 \stackrel{\circ}{R}+4 \hat{R}] d M .
\end{aligned}
$$

$$
\chi(M)=\frac{1}{384 \pi^{3}} \int_{M}\left[\frac{1}{9} S^{3}-S|R|^{2}-8 \stackrel{\circ}{R}+4 \hat{R}\right] d M .
$$

Therefore (3.4) is obtained from (3.5) and (3.1).

We now proceed to prove the theorem. We need the asymptotic expansion of Minakshisundaram-Pleijel for $\operatorname{Spec}(M, g)$ given by

$$
\sum_{k} \exp \left(\lambda_{k} t\right) \sim(4 \pi t)^{-m / 2}\left[a_{0}+a_{1} t+a_{2} t^{2}+\cdots\right],
$$

where $m=\operatorname{dim} M$. The coefficients $a_{0}, a_{1}, a_{2}$ and $a_{3}$ have been computed by Berger [1] and Sakai [6]:

$$
\begin{aligned}
& a_{0}=\operatorname{Vol}(M), \\
& a_{1}=\frac{1}{6} \int_{M} S d M, \\
& a_{2}=\frac{1}{360} \int_{M}\left[5 S^{2}-2\left|R_{1}\right|^{2}+2|R|^{2}\right] d M, \\
& a_{3}=\frac{1}{6 !} \int_{M}\left[-\frac{142}{63}|\nabla S|^{2}-\frac{26}{63}\left|\nabla R_{1}\right|^{2}-\frac{1}{9}|\nabla R|^{2}+\frac{5}{9} S^{3}\right.
\end{aligned}
$$




$$
\begin{aligned}
& -\frac{2}{3} S\left|R_{1}\right|^{2}+\frac{2}{3} S|R|^{2}-\frac{4}{7} R^{a b} R_{b}^{c} R_{a c} \\
& +\frac{20}{63} R^{a b} R^{c d} R_{a c b d}-\frac{8}{63} R^{u v} R_{u}^{a b c} R_{v a b c} \\
& \left.+\frac{8}{21} \hat{R}\right] d M .
\end{aligned}
$$

It may be noticed that instead of $\operatorname{Spec}(M, g)=\operatorname{Spec}\left(M^{\prime}, g^{\prime}\right)$, we mainly use $a_{i}=a_{\imath}{ }^{\prime}$ for $\imath=0,1,2,3$.

Since the 6-dimensional non-Kähler $K$-space is an Einsteinian, the coefficients $a_{\imath}$ are rewritten

$$
\begin{aligned}
a_{0}= & \operatorname{Vol}(M), \\
a_{1}= & \frac{1}{6} S \operatorname{Vol}(M), \\
a_{2}= & \frac{7}{3 \cdot 180} S^{2} \operatorname{Vol}(M)+\frac{1}{180} \int_{M}|R|^{2} d M, \\
a_{3}= & \frac{248}{7 ! \cdot 3^{4}} S^{3} \operatorname{Vol}(M)+\frac{122}{7 ! \cdot 3^{3}} S \int_{M}|R|^{2} d M \\
& +\frac{1}{5 ! \cdot 3^{2}} \int_{M}\left[\frac{4}{7} \hat{R}-\frac{1}{6}|\nabla R|^{2}\right] d M .
\end{aligned}
$$

From these, $a_{\imath}=a_{\imath}{ }^{\prime}$ imply

$$
\begin{gathered}
\operatorname{Vol}(M)=\operatorname{Vol}\left(M^{\prime}\right), \\
S=S^{\prime}, \\
\int_{M}|R|^{2} d M=\int_{M^{\prime}}\left|R^{\prime}\right|^{2} d M^{\prime}, \\
\int_{M}\left[\frac{24}{7} \hat{R}-|\nabla R|^{2}\right] d M=\int_{M^{\prime}}\left[\frac{24}{7}-\hat{R}^{\prime}-\left|\nabla^{\prime} R^{\prime}\right|^{2}\right] d M^{\prime} .
\end{gathered}
$$

By Lemma 3.2, we have

$$
\begin{aligned}
& \int_{M}\left[\frac{24}{7} \hat{R}-|\nabla R|^{2}\right] d M \\
& =\frac{384 \cdot 4}{7} \pi^{3} \chi(M)+\frac{1}{7} \int_{M}\left[|\nabla R|^{2}-\frac{1}{15} S\left(|R|^{2}-\frac{1}{15} S^{2}\right)\right] d M \\
& \quad+\frac{101}{3 \cdot 5 \cdot 7} S \int_{M}|R|^{2} d M-\frac{101}{3^{2} \cdot 5^{2} \cdot 7} S^{3} \operatorname{Vol}(M) .
\end{aligned}
$$

Considering $(3.10) \sim(3.12)$ and Theorem $2.2, \chi(M)=\chi\left(M^{\prime}\right)$ implies 


$$
\int_{M}|L|^{2} d M=\int_{M}\left|L^{\prime}\right|^{2} d M^{\prime}
$$

Theorem B now follows from Theorem 2.4.

In the course of the proof, we established the following

Corollary 3.3. Let $(M, g, J)$ and $\left(M^{\prime}, g^{\prime}, J^{\prime}\right)$ be 6-dimensional complete and connected K-spaces which are non-Kählerian. We assume that $\operatorname{Spec}(M, g)=$ $\operatorname{Spec}\left(M^{\prime}, g^{\prime}\right) . \quad$ If

$$
\int_{M} \hat{R} d M=\int_{M^{\prime}} \hat{R}^{\prime} d M^{\prime} \quad \text { or } \int_{M} \stackrel{\circ}{R} d M=\int_{M^{\prime}} \stackrel{\circ}{R}^{\prime} d M^{\prime}
$$

is satısfied, then $(M, g)$ is Riemannian locally 3-symmetric if and only if $\left(M^{\prime}, g^{\prime}\right)$ is Riemannian locally 3-symmetric.

We shall conclude this paper by noticing the following

Proposition 3.4. Let $(M, g, J)$ be a 6-dimensional complete and connected $K$-space whrch is non-Kählerian. Then we have

$$
\chi(M) \leqq \frac{1}{64 \pi^{3}} \int_{M}\left[\hat{R}-\frac{3}{10} S\left(|R|^{2}-\frac{1}{15} S^{2}\right)\right] d M
$$

with equality holding if and only if $(M, g)$ is a Riemannian locally 3-symmetruc space.

Proof. By Lemma 3.2,

$$
\int_{M}|\nabla R|^{2} d M=-192 \pi^{3} \chi(M)+\frac{1}{2} \int_{M}\left[6 \hat{R}+\frac{1}{9} S^{3}-\frac{5}{3} S|R|^{2}\right] d M .
$$

From this and Theorem 2.2, we have

$$
\begin{aligned}
\int_{M}|L|^{2} d M & =-192 \pi^{3} \chi(M)+3 \int_{M}\left[\hat{R}-\frac{3}{10} S\left(|R|^{2}-\frac{1}{15} S^{2}\right)\right] d M \\
& \geqq 0 .
\end{aligned}
$$

\section{REFERENCES}

[1] M. Berger, Le spectre des variétés riemanniennes, Rev. Roumaine Math. Pures Appl. 13 (1968), 915-931.

[2] A. Frölicher, Zur Differentialgeometrie der komplexen Strukturen, Math. Ann. 129 (1955), 50-95.

[3] T. Fukami and S. Ishihara, Almost Hermitian structure on $\mathrm{S}^{6}$, Tôhoku Math. J. 7 (1955), 151-156.

[4] A. GRAY, Riemannian manifolds with geodesic symmetries of order 3, J. Differential Geometry 7 (1972), 343-369. 
[5] M. Matsumoto, On 6-dimensional almost Tachibana spaces, Tensor, N.S. 23 (1972), 250-252.

[6] T. SAKAI, On eigen-values of Laplacian and curvature of Riemannian manifold, Tôhoku Math. J. 23 (1971), 589-603.

[7] T. Sato, Riemannian 3-symmetric spaces and homogeneous $K$-spaces, Mem. Fac. Tech. Kanazawa Univ. 12 (1979), 137-143.

[8] K. Sekigawa, Notes on homogeneous almost Hermitian manifolds, Hokkaldo Math. J. 7 (1978), 206-213.

[9] K. Sekigawa, Almost Hermitian manifolds satisfying some curvature conditions, kodai Math. J. 2 (1979), 384-405.

KANAZAWA UNIVERSITY 